\title{
Dynamic Gill and Mucous Microbiomes Track an Amoebic Gill Disease Episode in Farmed Atlantic Salmon
}

\section{Victor Blasco Birlanga}

National University of Ireland - Galway

Grace McCormack

National University of Ireland Galway

Umer Zeeshan ljaz

University of Glasgow

\section{Eugene McCarthy}

Galway Mayo Institute of Technology

\section{Cindy Smith}

University of Glasgow

Gavin Collins ( $\nabla$ gavin.collins@nuigalway.ie )

https://orcid.org/0000-0002-9947-1130

\section{Research Article}

Keywords: Alpha diversity, amoebic gill disease, Atlantic salmon, beta diversity, microbiome, PCR

Posted Date: May 21st, 2020

DOI: https://doi.org/10.21203/rs.3.rs-29747/v1

License: () (1) This work is licensed under a Creative Commons Attribution 4.0 International License. Read Full License 


\section{Abstract}

Background Amongst gill disorders in Atlantic salmon, amoebic gill disease (AGD) is currently one of the most common and virulent, resulting in large losses for the aquaculture industry. However, our understanding of the role of the gill microbiome during AGD development is limited. Thus, we undertook a longitudinal study with the main objective of characterising the microbiome of gill, and mucous, samples from farmed Atlantic salmon before, and during, an AGD episode. Using a newly optimised DNA extraction protocol, we sequenced rRNA genes from 90 Atlantic salmon gill microbiomes from a fish farm (West coast of Ireland) over the course of a summer season. The first aetiological agent of AGD, Neoparamoeba perurans, was quantified using PCR targeting 18S rRNA genes. The same analyses were done using mucous samples as suitable, non-lethal alternatives to gill samples. Microbiome features across the sampling campaign were distinguished, focusing on patterns before and during the AGD episode.

Results The richness and balance of the prokaryotic community on gills were trending upwards prior to the first appearance of AGD symptoms. The microbiome changed significantly, with reduced diversity and balance, after the AGD episode was confirmed, and the changing bacterial community was driven by competitive exclusion. However, this trend was reversed with the application of a first, and a second, freshwater bath treatment. Mucous samples behaved similarly. The variance of the entire prokaryotic community from both gill and mucous samples was significantly affected by the abundance of $\mathrm{N}$. perurans . Rubritalea sp. were abundant in every gill and mucous sample; however, other genera ( Dyadobacter, Shewanella and Pedobacter ) were maximally abundant in gill and mucous samples 12 days prior to the first detection of AGD symptoms.

Conclusions Gill and mucous microbiomes changed significantly after the first AGD symptoms were evident, correlating with $\mathrm{N}$. perurans concentrations and supporting a connection between the development of the AGD and the gill microbiome. Those changes, however, were reversed by the application of multiple freshwater treatments, which returned gills to a more healthy state. Despite differences between microbiome features from gill and mucous samples, the data establish mucous scrapings as suitable, non-lethal substitutes for partial characterisation of the whole prokaryotic community from fish gills. The genus Shewanella was widely present, and significantly more abundant, immediately before the first AGD symptoms than during the AGD episode, marking this out as a feasible, putative target in identifying proxies for early detection of AGD.

\section{Background}

Global fish production was 171 million tonnes in 2016, of which aquaculture represented 47\% [1] and approximately 2.2 million tonnes were production of Atlantic salmon (Salmo salar L.) [2]. The average annual growth of worldwide fish consumption was 3.2\% between 1961 and 2016, which was twice the rate of human population growth and significantly more than the growth in meat consumption [1]. 
However, the aquaculture industry faces several production challenges, including those related to infectious diseases. In particular, rates of gill disorders in salmon have increased in many countries [3], resulting in significant burdens on the sector due to lower growth rates, and increased susceptibility to other pathogenic agents and mortality. Amoebic gill disease (AGD) is currently one of the most important among the gill pathologies $[4,5]$, resulting in large losses for the industry [6].

The most prevalent symptoms of AGD include uncontrolled growth of fish gill cells (hyperplasia), overproduction of gill mucous, respiratory distress, and, in some cases, fish death, mainly due to reduced gas exchange [7]. As of yet, the only means to reduce AGD is to control the abundance of the first aetiological agent, the micro-eukaryote Neoparamoeba perurans [8], with, for example, the application of freshwater baths [4]. The clearest sign of $N$. perurans infection is the presence of white patches on gill tissue, allowing classification of gill health using an AGD score describing the extent of the patches and thus the disease [9]. In addition, a suite of molecular diagnostic tools is now available $[8,10]$, which allow the aetiological agent to be directly targeted from community DNA from samples of water or gill tissue. However, and despite inherent sensitivity and specificity, quantitative-PCR assays targeting N. perurans are only useful in confirming AGD episodes when already at an advanced stage but are ineffective as tools to predict outbreaks. Indeed, our understanding of several aspects of AGD and similar gill disorders is incomplete, including regarding the density of parasites and pathogens required to provoke symptoms; the involvement of other microorganisms, such as viruses, bacteria and zooplankton; stock genetics; and environmental parameters [11].

It is well documented that the microbiome of plant and animal tissue plays important potential roles in several diseases of various macro-eukaryotes, variously affecting the severity of symptoms $[12,13,14$, $15,16]$. Indeed, fish are known to harbour natural microflora [17] that play a role in host defenses against pathogens [4]. The impact of AGD infection on the microbiome of the salmon gill, and vice versa, is currently unknown. To date, only one study has reported on AGD and associated bacteria [18], identifying the presence of Psychroserpens sp. only in AGD-diseased fish. However, that study was constrained by the relatively low-throughput nature of the culture-independent approaches available at that time. Thus, our understanding of the role of the gill microbiome in salmon gill health, and how it responds during AGD outbreaks, is limited. We hypothesise that the gill microbiome may influence the onset and development of AGD. Equally, we hypothesise that specific bacterial taxa will be associated with AGD and may, in turn, be useful in predicting probable AGD outbreaks in fish stocks.

We undertook a longitudinal study with the main objective of characterising the gill microbiome of farmed Atlantic salmon (Salmo salarL.), and to quantify $N$. perurans markers, before, and during, an AGD episode.

To achieve this, we also pursued two methodological objectives. First, we needed to develop an approach to reliably sample the gill microbiome. Although several previous studies have described extracting nucleic acids from, and applying molecular tools to study, gill tissue $[11,19,20]$, typically only partial gills (sometimes less than $10 \%$ total gill arch weight) have been used as samples. Equally, there are few data 
available on the efficiency of gill microbiome DNA recovery techniques. To avoid extrapolation of results from partial gill tissues to the whole gill microbiome, as well as under-sampling of-and underrepresentation of taxa in-the microbiome, we first undertook to optimise the extraction based on sampling of entire gill arches.

Finally, as gill sampling is inherently invasive, a non-lethal and less invasive sampling approach would provide the basis for more attractive monitoring tools. Thus, our second methodological objective was to test the efficacy of mucous sampling as a suitable alternative source to gills for AGD microbiome analyses.

\section{Results}

\section{Representative nucleic acids extraction method from fish gills}

The number of prokaryotic cells in the liquid phase increased across the first five of the successive washes. However, significantly fewer cells were counted after the sixth and seventh washes, suggesting that five such washes sufficiently recovered the majority of the prokaryotic cells on the gills (Fig. 1).. Alpha and beta-diversity (supported by PERMANOVA p $>0.05$ ) were not significantly different between each wash, similarly resolving the prokaryotic communities (Fig. S1).. Rarefaction curves (Fig. S2) indicated DNA sequencing was close to the saturation limit (or plateau) in each of the separate washes after host DNA removal ( 75\% of the reads).

The second step of the DNA extraction procedure (i.e. the precipitation step) resulted in a four-fold increase in DNA recovery (Fig. 1).. The average DNA recovery from gill samples was $3 \mathrm{ng} \mathrm{DNA} / \mathrm{mg}$ gill tissue.

Quantitative PCR assays indicated abundant amoeba along the washes (Fig. 1).. Although the concentration of $N$. perurans $18 \mathrm{~S}$ rRNA genes was relatively low after the fifth wash, the abundance was higher again after the sixth and seventh washes.

\section{Fish features and appearance of the AGD}

The length and weight of the salmon cohort increased progressively over the sampling campaign (Fig. 2) but had increased significantly by the final sampling point (T6). Based on AGD scoring, no clear symptoms were apparent until T3 (Fig. 2).. Equally, no N. perurans 18S rRNA genes were detected in any of the gill samples from any of the cages before T3 (Fig. 3).. N. perurans was detected in two samples at T2 but only after 40 PCR amplification cycles; based on this, and the AGD scores, both fish were considered to be AGD-negative. The first AGD symptoms on gills were detected one week before T3. Considering this, the farm decided to apply freshwater baths to the salmon stocks between T3 and T4, and between $\mathrm{T} 5$ and $\mathrm{T} 6$. 


\section{Impact of AGD, and freshwater baths, on salmon gill microbiome}

The richness and Shannon index of the prokaryotic community on gills increased between T0 and T3 (Fig. $4 a, b)$.. By T3, with the first symptoms of AGD, and the prevalence of $N$. perurans, the microbiome changed significantly toward reduced diversity and lower NRI (environmental filtering). However, this was reversed with the application of the first (between T3 and T4) and second (between T5 and T6) freshwater baths.

Beta-diversity analyses (Fig. 4C) using the Bray-Curtis metric indicated the gill microbiome of pre-smolted salmon was completely different to the community on gills of post-smolted salmon. This is consistent with beta-diversity using UniFrac and Weighted UniFrac metrics. Once all salmon were transferred to sea water, the bacterial community did not sharply change until the first AGD symptoms were detected by T3. The gill microbiomes on T5 and T6 separately clustered from the earlier samples.

The genus Rubritalea was found to be consistently present in gill microbiomes (Fig. $4 d$ ).. Some of the other genera found, including C. Branchiomonas cisticola, Tenacibaculum maritimum, Piscirickettsia salmonis, Piscichlamydia sp., Psychroserpens sp. and C. Fritschea sp., were also found in Atlantic salmon infected with different diseases and described as opportunistic bacteria (Table S1)..

Based on SPLS-DA analyses of discriminating genera (Fig. S4e), samples from the same time point clustered together, except those from T1 and T2. Some genera (e.g., Albidiferax, Pedobacter, Dyadobacter and Shewanella) were maximally abundant immediately prior to the onset of AGD (at T2 and almost two weeks before the first AGD symptoms were detected), which was not observable at any of the remaining timepoints.

\section{Feasibility of non-lethal mucous sampling to approximate gill microbiomes}

The alpha-diversity profiles of gill and mucous microbiomes were similar before the first appearance of AGD (Fig. 5a).. Shannon diversity indices of gill and mucous samples deviated significantly after the first detection of symptoms (T3), and mucous microbiomes were significantly less balanced than gill microbiomes at both $\mathrm{T} 3$ and $\mathrm{T} 4$.

No distinguishable patterns were apparent in gill or mucous samples using environmental filtering or beta-diversity analyses (Fig. 5b, c).. The genus Rubritalea, as well as a variety of opportunistic bacterial pathogens, which were apparent in gill samples (Table S1),, were also found in all corresponding mucous samples (Fig. 5d).. Microbiome variability in both gill and mucous samples was significantly affected by both time (23\%) and sample type (mucous or gill; $3 \%$ ), and mucous samples appeared to provide at least a partial characterisation of corresponding gill microbiomes (Fig. 5c).. 


\section{Sources of variation in N. perurans abundance}

Temperature and sample type (gill or mucous) were strongly related to $N$. perurans abundance (Table S2).. Contrary to conventional evidence (e.g., [4]), water temperature was negatively associated with the abundance of the amoeba. On the other hand, there was a significantly higher chance to detect more abundant $N$. perurans from mucous samples than from gill samples. No relationship was apparent between fish features (i.e. weight and length, or AGD score) and $N$. perurans abundance. Negative associations were observed between the abundance of $N$. perurans and salinity, oxygen concentrations and water clarity.

\section{Influence of N. perurans, and environmental factors, on the gill microbiome}

A subset of nine OTUs could explain 92\% of dissimilarity (Bray-Curtis distance) between gill samples (Table S1).. Time could explain $35 \%$ of variability amongst all gill OTUs in the study but only $20 \%$ of variability in this OTU sub-set. Similarly, N. perurans abundance could explain $5 \%$ of variability across all OTUs but only $3.7 \%$ in those nine OTUs.

Observing only data from gill microbiomes, only one group-Stentrophomonas sp.-was correlated (negatively) with any of the environmental metadata (water clarity) (Table S1)..

Considering both mucous and gill samples, a sub-set of 12 OTUs accounted for $91 \%$ of all dissimilarities across all OTUs (Table S1).. In this case, sample type (mucous or gill) was also considered in PERMANOVA analyses. Time could explain $22 \%$ of variability amongst all OTUs but only $20 \%$ of variability in this sub-set. N. perurans abundance could explain $2.4 \%$ of variability in the sub-set.

Again, considering mucous and gill data collectively, four OTUs from both gill and mucous microbiomes correlated with environmental metadata, of which only two OTUs correlated with fish features. One OTUGardnerella sp. - was positively correlated in the gill dataset with AGD scores, but negatively correlated with fish weight in both gill and mucous datasets.

MINT analysis of gill and mucous samples to determine possible indicators of AGD susceptibility found Altererythrobacter, Dyadobacter, Shewanella and Pedobacter were maximally abundant on gills and in mucous samples at T2 (Fig. S5),, which was 12 days prior to the first detection of AGD symptoms and when qPCR detected fewest N. perurans (Fig. 3)..

\section{Discussion}

\section{Advantages of the extraction protocol and scope for improvement}


Representativeness in sampling has been widely addressed across different fields [21], and including in contaminants degradation [22], macroorganism ecology [23] and microbial ecology [24], since undersampling reduces the reliability of observed patterns. Microbial analyses from field samples normally indicate high spatial and temporal heterogenicity, making representativeness critical in microbial ecology. There is currently no evidence indicating how representative partial gill arches are of the overall gill microbiome. Previous studies, such as Steinum et al. [11] and Schmidt et al. [20], have characterised bacterial communities from partial gills, but the approach may underestimate diversity in the microbiome. Commonly used nucleic acids extraction kits limit the volume, or mass, of tissue in extraction preparations to normally between 30 and $100 \mathrm{mg}$, which would preclude the use of entire gill arches (which typically exceed $100 \mathrm{mg}$ ).

Although it is also possible to extract DNA and RNA from whole gills using in-house purification protocols [19], commercially available isolation kits offer more reproducibility and, usually, user-friendly workflows. In addition, nucleic acids extraction protocols commonly incorporate a tissue disintegration step with bead-beating [19], resulting in high relative concentrations of host DNA in final extracts. Host DNA competes with microbiome DNA for PCR amplification, and sequencing depth, obscuring patterns in microbial community analyses [26]. Thus, a step to allow for isolation, and concentration, of microbial cells from whole gill arches is desirable. The DNA and RNA extraction protocol presented in this study included this critical step prior to the use of a commercially available extraction kit. This ensured DNA extractions comprised representative nucleic acids of the salmon gill microbiome, and that all of the abundant bacterial taxa were included, by extracting DNA from five pooled gill washes. Although additional $N$. perurans could be detected after the fifth wash, the study was focused on the role of the prokaryotic microbiome in AGD, and the first step of the protocol was thus established as a series of five washes.

Salmon DNA was present in extractions and roughly three quarters of reads were from host genes. Nevertheless, after removing host DNA from the analyses, the saturation of the rarefaction curves from bacterial sequences indicated no significant loss of measured bacterial diversity despite host contamination. Nonetheless, competition from high concentrations of host DNA could inhibit amplification of prokaryotic targets. Further modifications to the proposed DNA extraction procedure could be considered, including to deplete as much host DNA as possible using, for instance, blocking primers [25], propidium monoazide [26] or methylation-based enrichments [27].

\section{Impact of AGD and freshwater bathing on the gill microbiome}

It was reasonable to consider that the AGD episode started between T2 (June) and T3 (July) as the first symptoms were detected in early July and continued until T6. Opportunistic bacteria associated with various gill diseases were prominent on gills during the AGD episode. Those included: C. Branchiomonas cisticola, Tenacibaculum maritimum, Piscirickettsia salmonis, Piscichlamydia sp., Psychroserpens sp. 
and C. Fritschea sp. (Table S1),, the presence of which on salmon gills during the AGD episode may have contributed to gill damage, increasing the severity of AGD, as was suggested previously by Steinum et al. [11]. Due to circumstances beyond the authors' control, it was not possible to continue sampling after T6, when AGD scores and qPCR data indicated the salmon population was still affected.

Principally, the study provided a temporal profile of the microbiome on Atlantic salmon gills by focusing emphasis on microbial diversity metrics. Prokaryotic richness and community balance on gills of presmolted salmon were the lowest across the dataset. The gill microbiome may follow a similar trajectory to the gut microbiome $[15,28]$, whereby an initial phase characterised by low diversity in the microbial community transitions, with changes in salmon lifestyle, toward a richer and balanced community. After smoltification, there was a marked change in prokaryotic diversity on salmon gills, preserving balance and richness. The shifting salinity during the smoltification, however, can change the gill microbiome, as described previously for skin microbiomes [29]. Nonetheless, the environmental filtering analysis in our study, which considers phylogenetic clustering as a cue for environmental pressure, suggests that changes in the prokaryotic microbiome on the pre-smolted salmon gills may have inherent stochasticity, driven by competition with other taxa.

The alpha diversity of the prokaryotic gill microbiome continued to increase after smoltification, as was also observed by Llewellyn et al. [15]. In addition, the environmental filtering analysis suggested the main environmental pressure was around T2 (which was also supported by beta-diversity analyses). However, 7 days after the salmon were first symptomatic (T3), the environmental pressure was eased, and the structure of the microbial communities appeared less deterministic, driven instead by a competitive exclusion principle (the ecological principle whereby community assembly is unhindered, and is driven by competition amongst taxa). Those trends were previously observed in the literature, albeit in connection with a different disease [30]. At the same time, $N$. perurans concentrations, determined by qPCR profiles, sharply increased by T3. Thus, it is likely that changes in the microbial community structure were due to the influence of AGD. The results give credence to the hypothesis that the development of AGD and the dynamic gill microbiome are related. Nevertheless, a laboratory trial including negative controls would be necessary to confirm this. Laboratory conditions, not constrained by the commercial decisions and schedule of a fish farm, would allow for controlled environmental parameters, and tightly scheduled freshwater baths and sampling.

After the first AGD-affected timepoint, freshwater treatments were applied (between T3 and T4; and between T5 and T6) and alpha diversity (including phylogenetic measures) increased (Fig. 4).. As such, the intervention appeared successful and the gill microbiomes reverted to a stable state allowing inhibition of pathogenic colonisation of the gill tissue [15]. Thus, freshwater treatment more profoundly affected microbial community assembly than other physico-chemical parameters.

An alternative scenario immediately after the freshwater treatment would see a less diverse gill microbiome prevailing, with fewer commensal bacteria. This would have two consequences: a short-term and a long-term one. The first is the immediate reduction of the inhibitory effect of commensal bacteria 
on pathogen colonisation, either passively or actively [15]. The second is a decelerated development of the vertebrate adaptive immune system [31] due to reduced gill bacterial loads that otherwise enhance this phenomenon. Both outcomes could elevate the AGD susceptibility of the salmon. However, as there is no specific treatment available against $N$. perurans, freshwater baths are still the most feasible option in AGD treatment.

\section{Comparing gill and mucous microbiomes}

None of the predominant bacterial taxa were found to be unique to either of the sample types. This was not unexpected, as the DNA extraction from gill samples could be considered as a DNA co-extraction from both mucous and gill tissue. The mucous on gills is likely to detach from the gill to the wash solution during the DNA extraction protocol; hence, communities can intermingle with more homogenised microbial community structures for the two samples types. Thus, our null hypothesis was that there is a degree of similarity in the bacterial communities from mucous and gill samples. However, when we performed diversity analysis, we found significant differences in Shannon entropy between gill and mucous samples at T3 and T4. One possible explanation is that the mucous on gills could act as an insulator, limiting the impact of environmental factors on the gill tissue and the underlying microbial community. Thus, the bacteria in mucous samples were likely better adapted to the changing environment, and both more resistant and resilient than taxa on gills. Nevertheless, bacterial communities on gill and mucous samples were equally affected by freshwater baths, which eventually increased diversity after each treatment.

Although some differences between gill and mucous samples were found, the similarity of the sample types with respect to prokaryotic diversity (Fig. 5) means mucous scrapings appear suitable as a nonlethal alternative for partial characterisation of the whole-gill prokaryotic microbiome.

Furthermore, three bacterial genera (Shewanella sp., Dyadobacter sp. and Pedobacter sp.) were most relatively abundant 12 days before the first AGD symptoms in gill and mucous samples (Fig. S6).. The genera were previously found on intestine, skin, eggs and gill microbiomes from various fish (Table S1).. Of these, Shewanella sp. were amongst the most abundant OTUs in this study and were widely detected on skin and gill microbiomes in the literature [11,32,33]. Since its abundance was affected by the onset of AGD, Shewanella sp. is a candidate marker (in either gill or mucous samples) for early detection of AGD. This should be investigated further.

\section{Freshwater bathing appears to regulate response of $\mathbf{N}$. perurans to environmental drivers}

The available literature on the life details of $N$. perurans is patchy, and the relationship with other microorganisms in distinct environments, such as fish gills, is still mostly unknown [4]. However, it is known that unicellular microeukaryotes, such as N. perurans, feed on other microorganisms, such as 
bacteria or archaea [34]. The gill microbiome, and the interaction with N. perurans, may thus shape the host's immunological machinery and response to infection [12]. As in the case of other fish pathologies associated with microbiomes [35], AGD is thus a multifactorial gill disease [4], requiring complex interactions between environmental factors (temperature, salinity, etc.), the fish microbiome, the host's metabolism, and pathogens.

Despite this, quantification of $N$. perurans, combined with AGD scoring, is the most reliable approach currently available to track the progress of the disease. Therefore, changes in $N$. perurans abundance on gills were used to find correlations explaining the development of AGD (Table S3).. N. perurans abundance profiles did not, however, reveal any significant correlations with AGD scores or any other fish features (e.g., weight or length) in any subset regression model. Such results could be explained by the application of, and interruption by, freshwater treatments during the sampling period. Whilst the size of individual fish continued to increase throughout the sampling campaign, the AGD outbreak impacted the stocks and the fish farm decided to apply a freshwater treatment. This reduced the abundance of $N$. perurans on gills (Fig. 3).. The possible introduction to the population, at T6, of salmon from another cohort might explain the sharp increase observed in fish size at the last timepoint (Fig. 2)..

On the other hand, $N$. perurans abundance appeared significantly correlated with environmental factors in most of the subset regression models (Table S3).. Breaking with convention [4], N. perurans abundance was negatively correlated with seawater temperature in many of the subset regression models. This unexpected result could be explained by the application of freshwater baths over the summer, which reduced $N$. perurans abundance on gills whilst the seawater temperature continued to increase. Importantly, the influence on N. perurans in this way, suggests freshwater baths are still the most viable intervention strategy to treat AGD.

Finally, N. perurans abundance was also significantly positively correlated with mucous samples in every subset regression model (Table S3).. This confirms it was more likely to quantitate more $N$. perurans in the mucous than in the gill samples, making non-lethal mucous sampling more reliable in targeting $N$. perurans from salmon gills.

\section{Sources of variation of the microbiome on salmon gills}

Microbiome subset analyses of gill and mucous samples found several positive and negative correlations between the most variable part of the prokaryotic community and the environmental factors considered (Table S2).. Nevertheless, only one bacterial taxon correlated with fish features. Gardnerella sp. significantly, positively correlated with the AGD score, but negatively correlated with salmon weight. The results suggest those bacteria typically appear on less developed prokaryotic communities from immature salmon, or from AGD-affected salmon. The influence of freshwater treatments on those correlations was not clear. 
Several intrinsic and extrinsic sources of variation shape microbial communities, from study design to environmental factors. Environmental parameters explained for $31 \%$ of the variability in community structure (Table S2). N. perurans explained $5 \%$ of the variability in the whole prokaryotic community from gills and mucous. Thus, the connection between the development of AGD ( $N$. perurans quantification) and the prokaryotic microbiome on farmed Atlantic salmon gills was significant, despite the apparent impact of the freshwater treatments.

Furthermore, N. perurans abundance significantly correlated with subsets of the microbiome (Table S2).. It is likely that taxa, including Lactobacillus, Turicibacter, Stentrophomonas, Bifidobacterium, Allobaculum, Clostridium and Stentrophomonas, play a role in $N$. perurans predominance on the salmon gills. However, further studies will be required to better understand the relationships between the bacterial microbiome and AGD on salmon gills. Metagenomics of the prokaryotic community on AGD-affected gills, combined with $N$. perurans transcriptomics, would further elucidate the relationship.

\section{Conclusions}

In this study, a new procedure was optimised to representatively recover DNA from the microbiome of fish gills. Applying this optimised procedure, we were able to determine that, after the onset of the AGD episode, the prokaryotic community on salmon gills shifted toward lower diversity and less balance. In addition, the possible correlation between the development of AGD ( $N$. perurans abundance) and the prokaryotic microbiome from gill and mucous samples was confirmed, explaining $5 \%$ of the bacterial community variability. Declining bacterial diversity was reversed after freshwater treatments, increasing the richness and community balance.

Three different bacteria were found to be maximally relatively abundant on gills and mucous samples 12 days before first AGD symptoms appeared. Among them, Shewanella, appeared to be in the top 25 most abundant OTUs, indicating this genus as a possible target for early AGD prediction assays. Bacterial communities from gill and mucous samples from this study presented very close similarities, establishing mucous scrapings as a suitable non-lethal substitute for gill sampling for partial characterisation of the whole-gill prokaryotic community.

Combined laboratory and field trials would allow for more complete understanding of the connection between AGD and the gill microbiome. Nonetheless, the results point toward the potential for earlier, more efficient, and less invasive diagnoses and treatment of AGD.

\section{Materials And Methods}

\section{Longitudinal Atlantic Salmon Gill Microbiome Sampling}

Salmon gill samples were collected from a fish farm in Bertraghboi Bay (western coast of Ireland; $53^{\circ} 22^{\prime} 03.1^{\prime \prime} \mathrm{N}, 9^{\circ} 51^{\prime} 47.5^{\prime \prime} \mathrm{W}$ ), with the collaboration of MOWI Ireland. Sampling was started in Spring (T0, May 2017) using freshwater pre-smolts and followed the cohort for six months (T1, June-T6, October) in 
seawater cages (Fig. 6),, during and after the summer months, when AGD is more likely to appear (Oldham et al., 2016). At each of the seven time points (T0-T6), five salmon were sampled from each of three separate cages. The fish were then over-anesthetised (tricaine methanosulfonate MS-222, 200 $\mathrm{mg} / \mathrm{L}$ ). The weight and length of each fish was measured, and the AGD score was recorded following the guidelines of [9].

The gill microbiome was sampled as follows: first, the operculum was aseptically removed using sterile scissors; second, mucous was collected (at T2-T6) from the first brachial arch by scraping using a sterile spatula. Mucous was sampled in this way from each of the five fish from the respective cages and pooled in $200 \mu \mathrm{l} \mathrm{TE} \mathrm{buffer} \mathrm{(10} \mathrm{mM} \mathrm{Tris-Cl,} \mathrm{pH} \mathrm{7.5;} 1 \mathrm{mM}$ EDTA). Each of the three mucous pools (one from each cage) was then divided into two technical replicates for further processing $(n=6)$. The second brachial arch of each fish was then excised using sterile scissors and forceps, and placed in respective sterile, labelled, tubes prior to flash freezing $(n=15)$.

Water temperature $\left({ }^{\circ} \mathrm{C}\right)$, salinity $(\% \circ)$, dissolved oxygen $(\%)$ and turbidity (Secchi depth, metres), were each measured daily throughout the sampling campaign at a depth of $5 \mathrm{~m}$ using a ConductivityTemperature-Depth (CTD) measuring device (YSI 2030 device; ID: 12391).

\section{Optimisation of nucleic acids extraction from gills}

To extract the DNA from gill samples, a three-step procedure was developed. First, $50 \mu$ trypsin-EDTA ( $0.5 \%$ trypsin [w/v] in 6.8 mM EDTA; Sigma-Aldrich, Saint Louis, USA) were added along with $450 \mu \mathrm{l} 0.5 \mathrm{M}$ EDTA to a gill in a 2-ml microcentrifuge tube prior to sonication for 5 min (Kerry Ultrasonic Baths, model PUL-125). The gill was then removed to a fresh tube and the process was repeated multiple (six) times. The number of washes required to optimally detach bacterial cells was determined based on the concentration of prokaryotic cells in the liquid phase from each successive wash measured using a hemocytometer (10 central squares [0.20 mm x $0.20 \mathrm{~mm}$ ] were considered in each of three replicates for each wash).

Second, the liquid phase from each wash step was pooled in a 15-ml tube, and the gill was discarded. An equal volume of ice-cold isopropanol was added along with a 0.1 volume of $3 \mathrm{M}$ sodium acetate to precipitate free DNA, which was then recovered with microbial cells by centrifuging for $30 \mathrm{~min}$ at $>8,000$ $\mathrm{g}\left(4^{\circ} \mathrm{C}\right)$ to form three layers. The top and middle layers were discarded.

Third, an extraction kit (AllPrep DNA/RNA extraction kit, QIAGEN, Hilden, Germany) was used to recover nucleic acids from the bottom layer containing the pellet following the manufacturer's instructions without any modifications.

To determine the efficacy of the second step in enhancing DNA extraction efficiency, DNA recovery was compared with and without the precipitation stage. DNA yield was measured as ng DNA recovered per mg gill tissue, so as to ensure a fair comparison of DNA recovery across different gill weights. 


\section{DNA extraction from gills and mucous samples}

DNA was extracted from the gills at each of the seven timepoints $(n=105)$ according to the optimised protocol. Mucous samples (100 $\mu \mathrm{l}$ from the pooled mixtures) from each of T2-T6 $(n=30)$ were processed using the extraction kit (AllPrep DNA and RNA extraction kit, QIAGEN, Hilden, Germany) according to the manufacturer's instructions. Concentrations of double-stranded DNA were determined using a Qubit dsDNA BR Assay Kit (ThermoFisher Scientific, Paisley, UK), following the instructions provided.

\section{S rRNA gene sequencing and N. perurans 18S rRNA gene quantification}

The V4 and OutTS1-spanning regions of 16S rRNA genes from gill and mucous DNA samples were amplified using the oligonucleotide primers 515F (5'-GTGCCAGCMGCCGCGGTAA-3') and 806R (5'GGACTACHVGGGTWTCTAAT-3') [36]. PCR mixtures (25 $\mu$ final volume) contained: $12.5 \mathrm{ng}$ genomic DNA, $0.2 \mu \mathrm{M}$ (final concentration) of each primer (515F and 806R), and $12.5 \mu \mathrm{l} 2 \mathrm{X}$ KAPA HiFi Hot Start Ready Mix ( $0.25 \mathrm{U}$; Roche). The PCR comprised of initial denaturation at $95^{\circ} \mathrm{C}$ for $3 \mathrm{~min} ; 25$ cycles of $95^{\circ} \mathrm{C}$ for $30 \mathrm{~s}, 55^{\circ} \mathrm{C}$ for $30 \mathrm{~s}$ and $72^{\circ} \mathrm{C}$ for $30 \mathrm{~s}$; and a final incubation at $72^{\circ} \mathrm{C}$ for $5 \mathrm{~min}$. PCR products were purified (AMPure XP for PCR Purification, Beckman Coulter, USA) before adding Illumina sequencing adapters and indices using a Nextera XT Index Kit (Cambridge, United Kingdom) following the manufacturer's instructions. A second PCR purification was done before quantifying the DNA using a 2100 Bioanalyzer system (Agilent Technologies), normalising the DNA concentration (to $4 \mathrm{nM}$ ) and pooling the libraries with unique indices. Pooled libraries were denatured $(0.2 \mathrm{~N} \mathrm{NaOH})$, diluted and heatdenatured before sequencing. Amplicons were sequenced using an Illumina MiSeq platform [37] using $5 \%$ PhiXas as internal control, following Illumina's recommendations.

The concentration of $N$. perurans in gill and mucous samples was estimated in quantitative PCR assays targeting partial 18S rRNA gene sequences specific to the amoeba and using the primers NP1 (5'AAAAGACCATGCGATTCGTAAAGT-3') and NP2 (5'-CATTCTTTTCGGAGAGTGGAAATT-3'), with the NPP (6-FAM- ATCATGATTCACCATATGTT-MGB) probe, according to the procedure as described in detail by [10]. Each PCR mixture $(25 \mu \mathrm{l})$ contained $5 \mu \mathrm{l}$ microbial genomic DNA $(5 \mathrm{ng} / \mu \mathrm{l}), 12.5 \mu \mathrm{l} \mathrm{TaqMan}{ }^{\circledR}$ Universal 2 Master Mix (Applied Biosystems), and final concentrations of $300 \mathrm{nM}$ primer NP1, $900 \mathrm{nM}$ primer NP2 and $200 \mathrm{nM}$ probe NPP. Each qPCR program comprised of an initial denaturation at $95^{\circ} \mathrm{C}$ for $15 \mathrm{~min}$, followed by 45 cycles of $95^{\circ} \mathrm{C}$ for $15 \mathrm{~s}$ and $56^{\circ} \mathrm{C}$ for $30 \mathrm{~s}$ in a real-time PCR thermocycler (Applied Biosystems AB7500). Positive and negative controls were added into each run, as well as internal and external process controls [10].

\section{Bioinformatics and Statistical Analyses}


Operational taxonomic units (OTUs) were constructed at $97 \%$ similarity using the VSEARCH workflow after pre-filtering stages including the removal of host contamination. In the final analysis, 3,834 clean OTUs were extracted for $n=162$ samples on which multivariate statistical analyses were performed in $\mathrm{R}$ in view of the metadata collected in this study. The details of the bioinformatics steps, along with procedures for the statistical analyses of fish features, QPCR data and environmental parameters, as well as software and R packages used, are provided in the Supplementary Methods (Supplementary File)..

\section{List Of Abbreviations}

AGD: Amoebic Gill Disease.

DNA: Desoxiribonucleic Acid

PCR: Polymerase Chain Reaction

qPCR: quantitative Polymerase Chain Reaction

EDTA: Ethylenediaminetetraacetic Acid

OTU: Operational Taxonomic Unit

PERMANOVA: Permutational Analysis Of Variance

ANOVA: Analysis Of Variance

\section{Declarations}

\section{Ethics approval and consent to participate}

Fish samples were obtained from a commercial farm during routine monitoring, and so ethics approval was not required.

\section{Consent for publication}

All authors give their consent to publish the present research article.

\section{Availability of data and materials}

The sequencing data are available in the European Nucleotide Archive under the study accession number PRJEB32307 (http://www.ebi.ac.uk/ena/data/view/PRJEB32307), with further detail in the supplementary files. 


\section{Competing interests}

The authors declare that they have no competing interests.

\section{Funding}

This work was funded as part of the 'ADIOS' (grant award No. 15S745) by the Irish Department of Agriculture, Food and the Marine (Ireland). UZI was sup-ported by a NERC independent research fellowship (NERC NE/L011956/1).

\section{Authors' contributions}

\section{Affiliations}

Microbiology, School of Natural Sciences National University of Ireland Galway, University Road, Galway, H91 TK33, Ireland.

Victor B. Birlanga \& Gavin Collins.

Zoology, School of Natural Sciences National University of Ireland Galway, University Road, Galway, H91 TK33, Ireland.

Grace McCormack.

Ryan Institute, National University of Ireland Galway, University Road, Galway, H91 TK33, Ireland.

Grace McCormack \& Gavin Collins.

Infrastructure and Environment, School of Engineering, University of Glasgow, Rankine Building, Oakfield Avenue, Glasgow, G12 8LT, United Kingdom.

Umer Z. ljaz \& Cindy Smith.

Institute of Science, Technology and Medicine, Galway-Mayo Institute of Technology, Galway, H91 T8NW, Ireland.

Eugene McCarthy.

Contributions

$\mathrm{CS}, \mathrm{GMC}$ and EMC conceived the idea underpinning the present study. VBB and GC conducted the field sampling. VBB did the DNA extractions and all other wet experimentation. VBB and UZI did the data 
analyses. VBB, UZI and GC wrote the initial manuscript with comments from CS, GMC and EMC. All authors read and approved the final manuscript.

\section{Acknowledgements}

The authors thank the fish farm operated by MOWI (in particular, Catherine McManus), and Neil Ruane (Marine Institute, Galway) for his help in N. peru-rans qPCRs. Ashlea Bryne is thanked for her contribution to protocol optimisa-tion, as are the remaining researchers on the 'ADIOS' at the Galway-Mayo In-stitute of Technology for their continuous flux of ideas and support.

\section{References}

1. Food and Agriculture Organization of the United Nations. Fisheries Department. The state of world fisheries and aquaculture. Food and Agriculture Organization of the United Nations. 2018:2.

2. Kontali Analyse Salmon World (2016) (www.kontali.com).

3. Rodger HD, Henry L, Mitchell SO. Non-infectious gill disorders of marine salmonid fish. Reviews in Fish Biology and Fisheries. 2011;21(3):423-40.

4. Oldham T, Rodger $H$, Nowak BF. Incidence and distribution of amoebic gill disease (AGD) - An epidemiological review. Aquaculture. 2016;457:35-42.

5. Marcos-López M, Ruiz CE, Rodger HD, O'Connor I, MacCarthy E, Esteban MÁ. Local and systemic humoral immune response in farmed Atlantic salmon (Salmo salar L.) under a natural amoebic gill disease outbreak. Fish \& shellfish immunology. 2017;66:207-16.

6. Rodger HD. Amoebic gill disease (AGD) in farmed salmon (Salmo salar) in Europe. Fish Veterinary Journal. 2014(14):16-27.

7. Munday BL, Zilberg D, Findlay V. Gill disease of marine fish caused by infection with Neoparamoeba pemaquidensis. Journal of Fish Diseases. 2001;24(9):497-507.

8. Young ND, Crosbie PB, Adams MB, Nowak BF, Morrison RN. Neoparamoeba perurans n. sp., an agent of amoebic gill disease of Atlantic salmon (Salmo salar). International journal for parasitology. 2007;37(13):1469-81.

9. Taylor RS, Muller WJ, Cook MT, Kube PD, Elliott NG. Gill observations in Atlantic salmon (Salmo salar, L.) during repeated amoebic gill disease (AGD) field exposure and survival challenge. Aquaculture. 2009;290(1-2):1-8.

10. Downes JK, Henshilwood K, Collins EM, Ryan A, Connor IO, Rodger HD, MacCarthy E, Ruane NM. A longitudinal study of amoebic gill disease on a marine Atlantic salmon farm utilising a real-time PCR 
assay for the detection of Neoparamoeba perurans. Aquaculture Environment Interactions. 2015;7(3):239-51.

11. Steinum T, Sjåstad K, Falk K, Kvellestad A, Colquhoun DJ. An RT PCR-DGGE survey of gill-associated bacteria in Norwegian seawater-reared Atlantic salmon suffering proliferative gill inflammation. Aquaculture. 2009;293(3-4):172-9.

12. Gómez GD, Balcázar JL. A review on the interactions between gut microbiota and innate immunity of fish. FEMS Immunology \& Medical Microbiology. 2008;52(2):145-54.

13. Berendsen RL, Pieterse $\mathrm{CM}$, Bakker PA. The rhizosphere microbiome and plant health. Trends in plant science. 2012;17(8):478-86.

14. Cho I, Blaser MJ. The human microbiome: at the interface of health and disease. Nature Reviews Genetics. 2012;13(4):260-70.

15. Llewellyn MS, Boutin S, Hoseinifar SH, Derome N. Teleost microbiomes: the state of the art in their characterization, manipulation and importance in aquaculture and fisheries. Frontiers in microbiology. 2014;5:207.

16. Voreades N, Kozil A, Weir TL. Diet and the development of the human intestinal microbiome. Frontiers in microbiology. 2014;5:494.

17. Smith CJ, Danilowicz BS, Meijer WG. Characterization of the bacterial community associated with the surface and mucus layer of whiting (Merlangius merlangus). FEMS microbiology ecology. 2007;62(1):90-7.

18. Bowman JP, Nowak B. Salmonid gill bacteria and their relationship to amoebic gill disease. Journal of Fish Diseases. 2004;27(8):483-92.

19. Lowrey L, Woodhams DC, Tacchi L, Salinas I. Topographical mapping of the rainbow trout (Oncorhynchus mykiss) microbiome reveals a diverse bacterial community with antifungal properties in the skin. Appl. Environ. Microbiol.. 2015;81(19):6915-25.

20. Schmidt V, Amaral-Zettler L, Davidson J, Summerfelt S, Good C. Influence of fishmeal-free diets on microbial communities in Atlantic salmon (Salmo salar) recirculation aquaculture systems. Appl. Environ. Microbiol. 2016;82(15):4470-81.

21. Paoletti C, Esbensen KH. Distributional assumptions in food and feed commodities-development of fit-for-purpose sampling protocols. Journal of AOAC International. 2015;98(2):295-300.

22. Peter A, Steinbach A, Liedl R, Ptak T, Michaelis W, Teutsch G. Assessing microbial degradation of oxylene at field-scale from the reduction in mass flow rate combined with compound-specific isotope analyses. Journal of contaminant hydrology. 2004;71(1-4):127-54. 
23. Dufrêne M, Legendre P. Species assemblages and indicator species: the need for a flexible asymmetrical approach. Ecological monographs. 1997;67(3):345-66.

24. Ranjard L, Lejon DP, Mougel C, Schehrer L, Merdinoglu D, Chaussod R. Sampling strategy in molecular microbial ecology: Influence of soil sample size on DNA fingerprinting analysis of fimgal and bacterial communities. Environmental Microbioloc. 2003;5(1):1.

25. Arenz BE, Schlatter DC, Bradeen JM, Kinkel LL. Blocking primers reduce co-amplification of plant DNA when studying bacterial endophyte communities. Journal of microbiological methods. 2015;117:1-3.

26. Marotz CA, Sanders JG, Zuniga C, Zaramela LS, Knight R, Zengler K. Improving saliva shotgun metagenomics by chemical host DNA depletion. Microbiome. 2018;6(1):42.

27. Chiou KL, Bergey CM. Methylation-based enrichment facilitates low-cost, noninvasive genomic scale sequencing of populations from feces. Scientific reports. 2018;8(1):1975.

28. Romero J, Navarrete P. $16 \mathrm{~S}$ rDNA-based analysis of dominant bacterial populations associated with early life stages of coho salmon (Oncorhynchus kisutch). Microbial ecology. 2006;51(4):422-30.

29. Lokesh J, Kiron V. Transition from freshwater to seawater reshapes the skin-associated microbiota of Atlantic salmon. Scientific reports. 2016;6:19707.

30. Das A, Pereira V, Saxena S, Ghosh TS, Anbumani D, Bag S, Das B, Nair GB, Abraham P, Mande SS. Gastric microbiome of Indian patients with Helicobacter pylori infection, and their interaction networks. Scientific reports. 2017;7(1):1-9.

31. Hooper LV, Littman DR, Macpherson AJ. Aj: M. Interactions between the Microbiota and the immune system. Science. 2012;336:1268-73.

32. Svanevik CS, Lunestad BT. Characterisation of the microbiota of Atlantic mackerel (Scomber scombrus). International Journal of Food Microbiology. 2011;151(2):164-70.

33. Brown RM, Wiens GD, Salinas I. Analysis of the gut and gill microbiome of resistant and susceptible lines of rainbow trout (Oncorhynchus mykiss). Fish \& shellfish immunology. 2019;86:497-506.

34. Thaler M, Lovejoy C. Distribution and diversity of a protist predator Cryothecomonas (Cercozoa) in Arctic marine waters. Journal of Eukaryotic Microbiology. 2012;59(4):291-9.

35. Talwar C, Nagar S, Lal R, Negi RK. Fish gut microbiome: current approaches and future perspectives. Indian journal of microbiology. 2018;58(4):397-414.

36. Caporaso JG, Lauber CL, Walters WA, Berg-Lyons D, Lozupone CA, Turnbaugh PJ, Fierer N, Knight R. Global patterns of $16 \mathrm{~S}$ rRNA diversity at a depth of millions of sequences per sample. Proceedings of 
the national academy of sciences. 2011;108(Supplement 1):4516-22.

37. Caporaso JG, Lauber CL, Walters WA, Berg-Lyons D, Huntley J, Fierer N, Owens SM, Betley J, Fraser L, Bauer M, Gormley N. Ultra-high-throughput microbial community analysis on the Illumina HiSeq and MiSeq platforms. The ISME journal. 2012;6(8):1621-4.

\section{Figures}

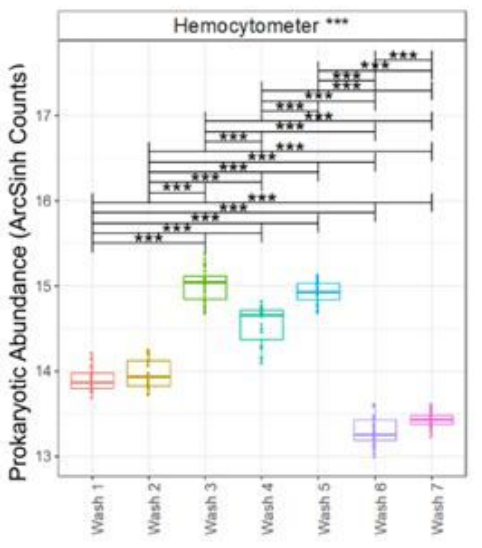

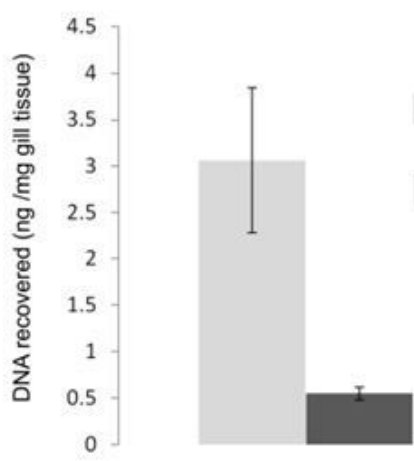

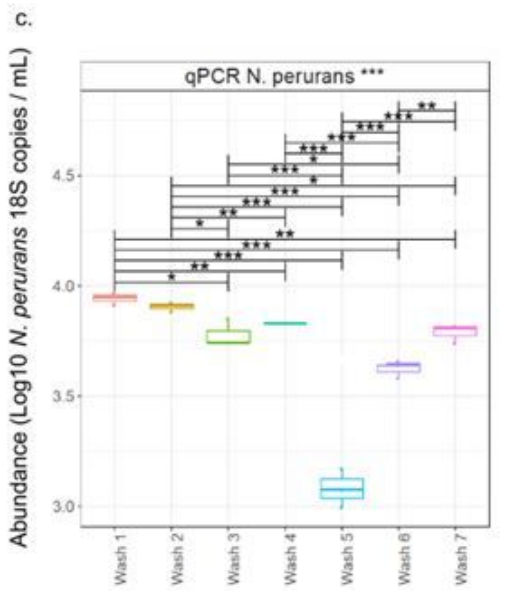

Figure 1

ANOVA of pair-wise comparison of extrinsic metadata considered in this study, and af-ter applying appropriate normalization (where necessary). Lines connect two categories where the differences were significant with * $(P<0.05)$, ** $(P<0.01)$, or *** $(p<0.001)$. (a) ArcSinh normalisation was used for prokaryote cells from each wash); (b) compari-son of DNA yield from extractions with and without isopropanol and acetic acid precipi-tation (replicate treatments); (c) qPCR data from each wash transformed using Log10 of the original results for N. perurans.
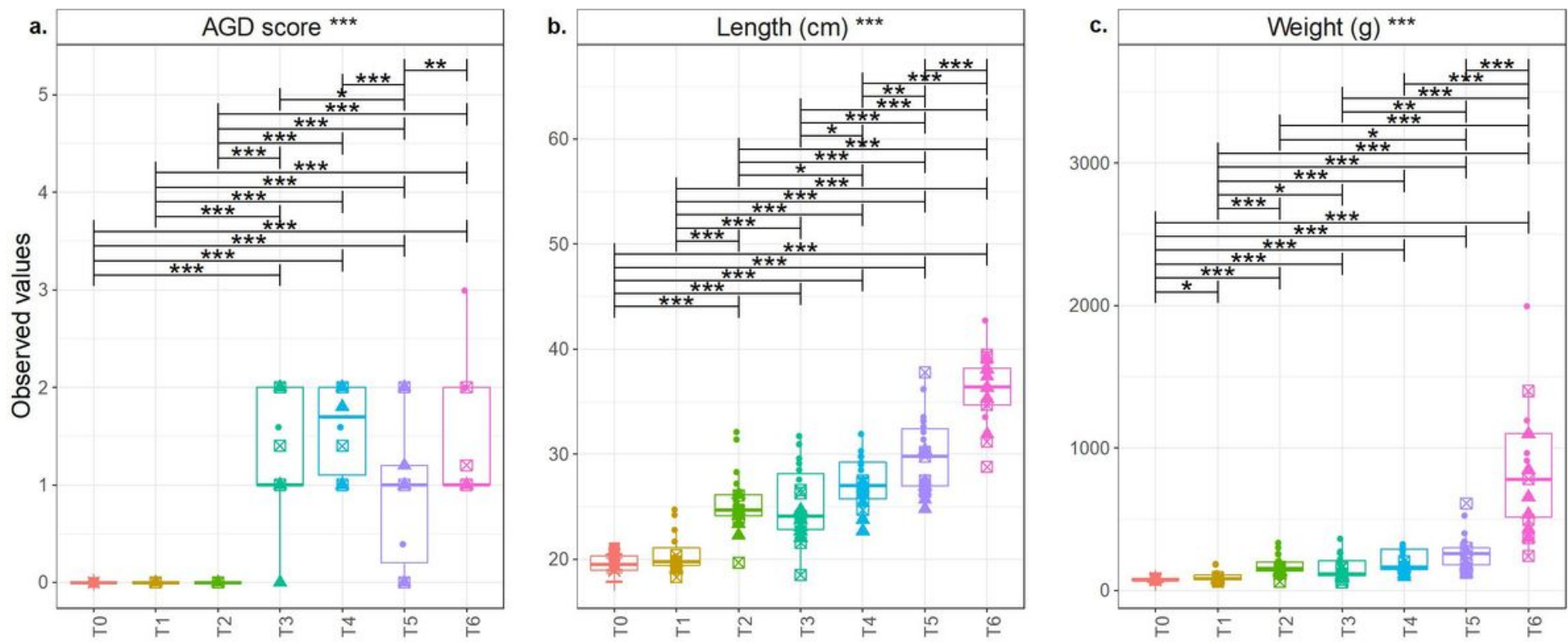
Figure 2

ANOVA of pair-wise comparison of extrinsic metadata considered in this study, and af-ter applying appropriate normalization (where necessary). Lines connect two categories where the differences were

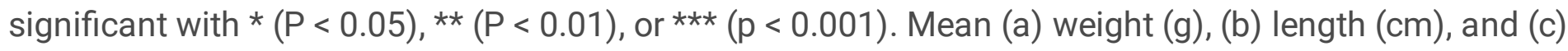
AGD score at each of the timepoints.

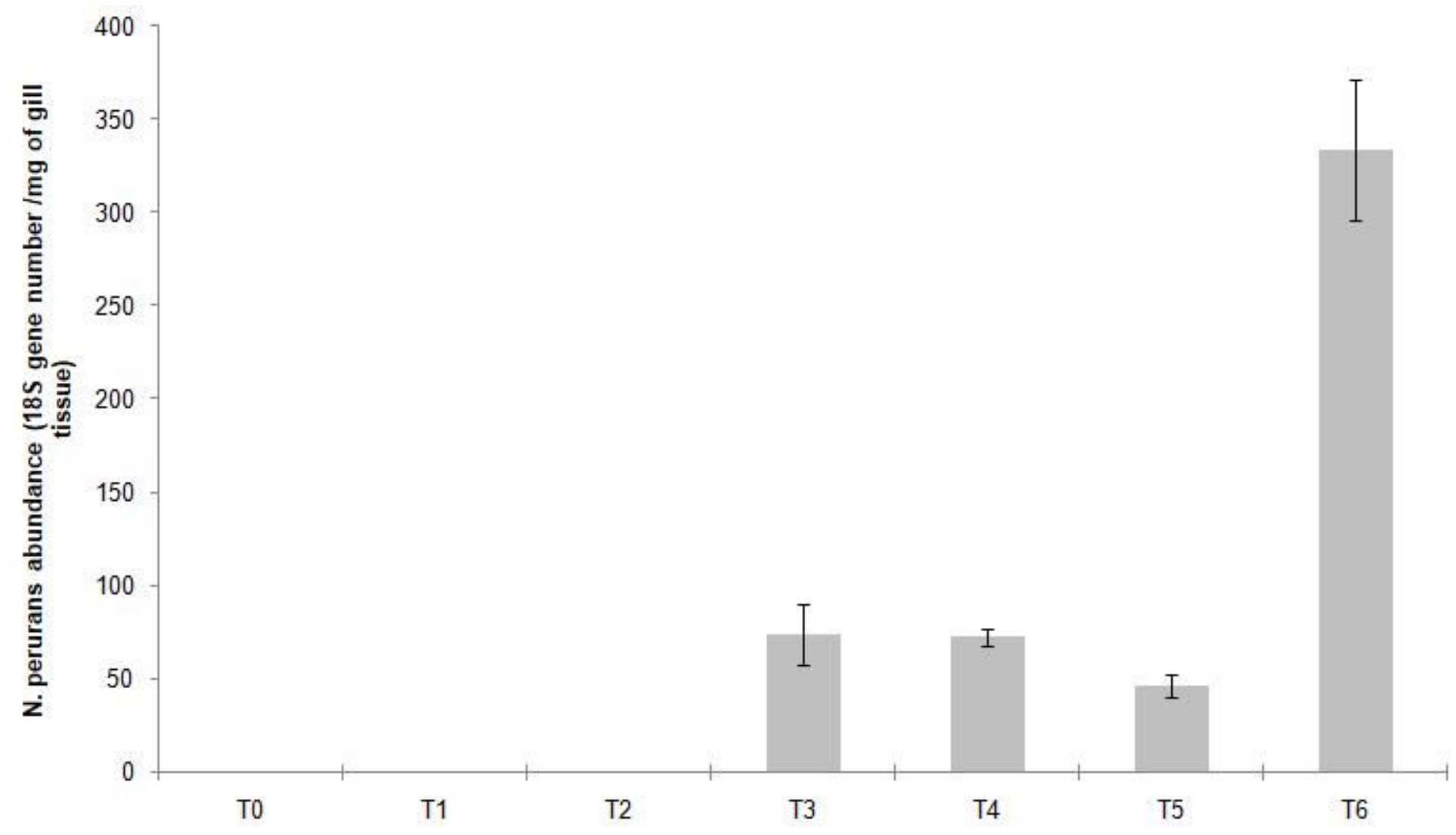

Figure 3

Concentrations of N. perurans 18 S rRNA genes in DNA extractions from salmon gills measured by qPCR assays. 
a. Alpha Diversity

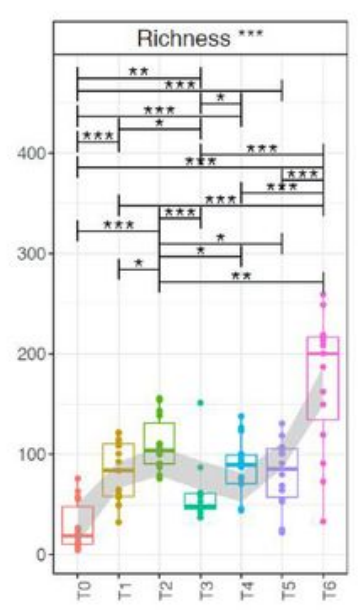

b. Environmental Filtering
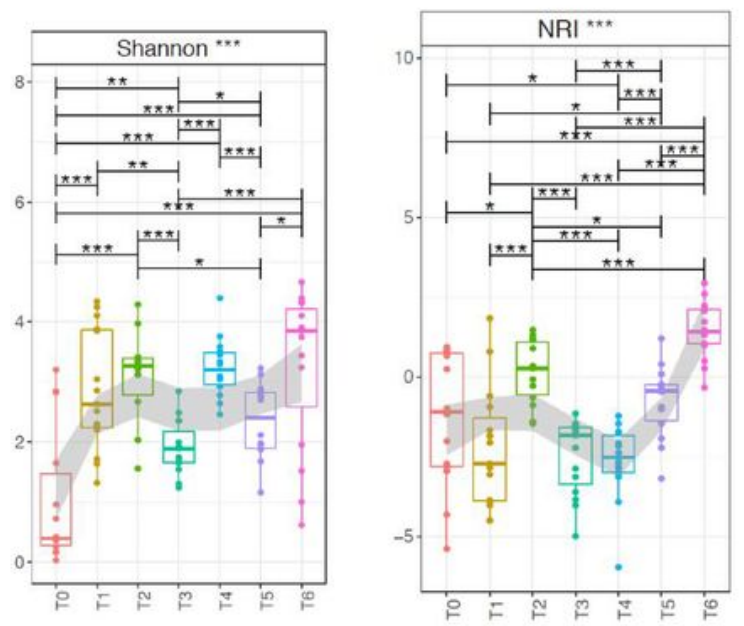

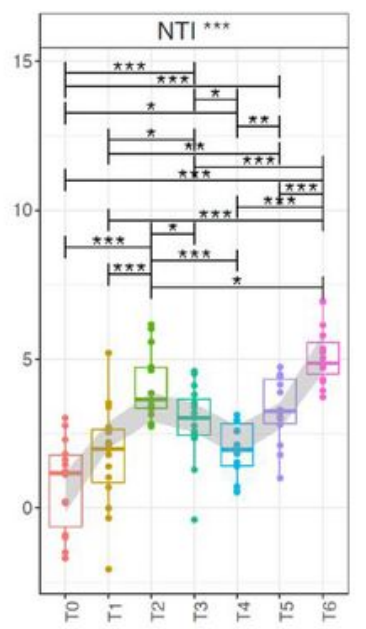

\section{Beta Diversity}

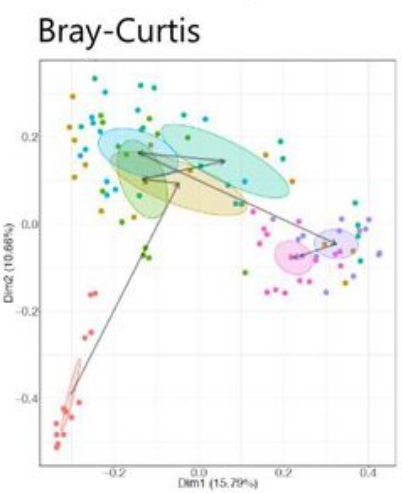

PERMANOVA: $R^{2}=0.35(p=0.001 \cdots)$

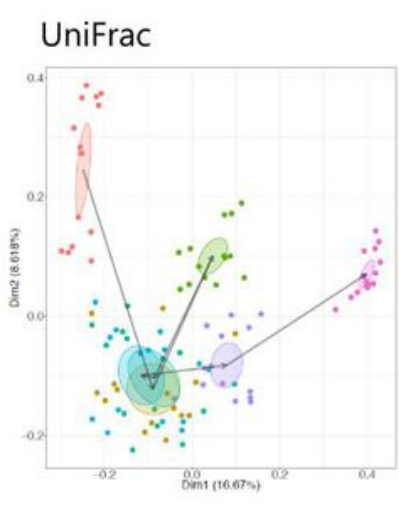

PERMANOVA: $R^{2}=0.35(p=0.001 \cdots)$

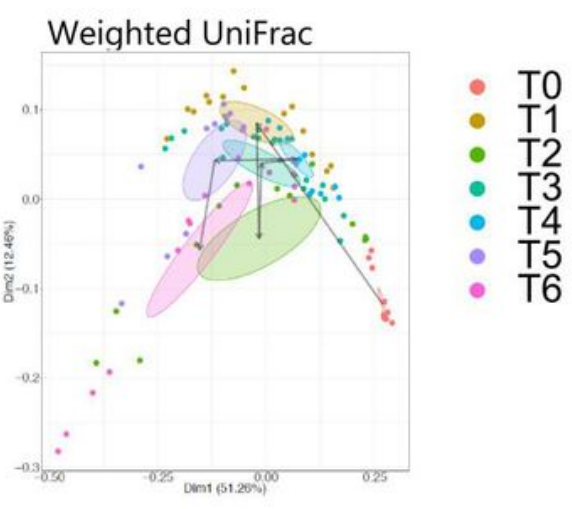

PERMANOVA: $R^{2}=0.43(p=0.001 \cdots)$

\section{d. Top 25 Abundant OTUs}

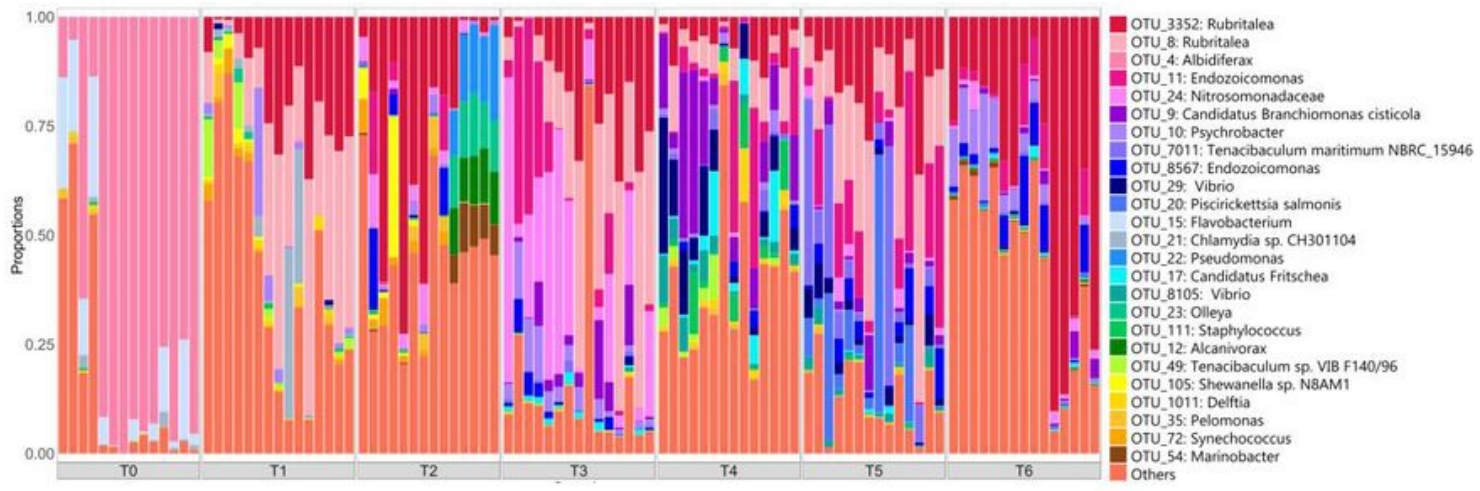

\section{Figure 4}

Microbial diversity and community structure from temporal gill analyses. (a), (b) and (c) represent alpha diversity, environmental filtering, and beta diversity indices, respective-ly. In (a) and (b), the regions have been shaded using LOESS smoothing in ge-om_smooth() when plotting using R'sggplot(). In (c), ellipses are drawn at 95\% confi-dence interval of standard error with lines from T0 to T6 plotted at the mean of the ordi-nation values at each timepoint. (d) shows community structure based on relative abundance of 
the top 25 most abundant OTUs from across each time point, where 'others' refers to all OTUs not included in the top 25; lines for figures (a) and (b) connect two categories where the differences were significant (ANOVA) with * $(P<0.05)$, ** $(P<0.01)$, or *** $(p<0.001)$.
a. Alpha Diversity
b. Environmental Filtering
Gill Mucous
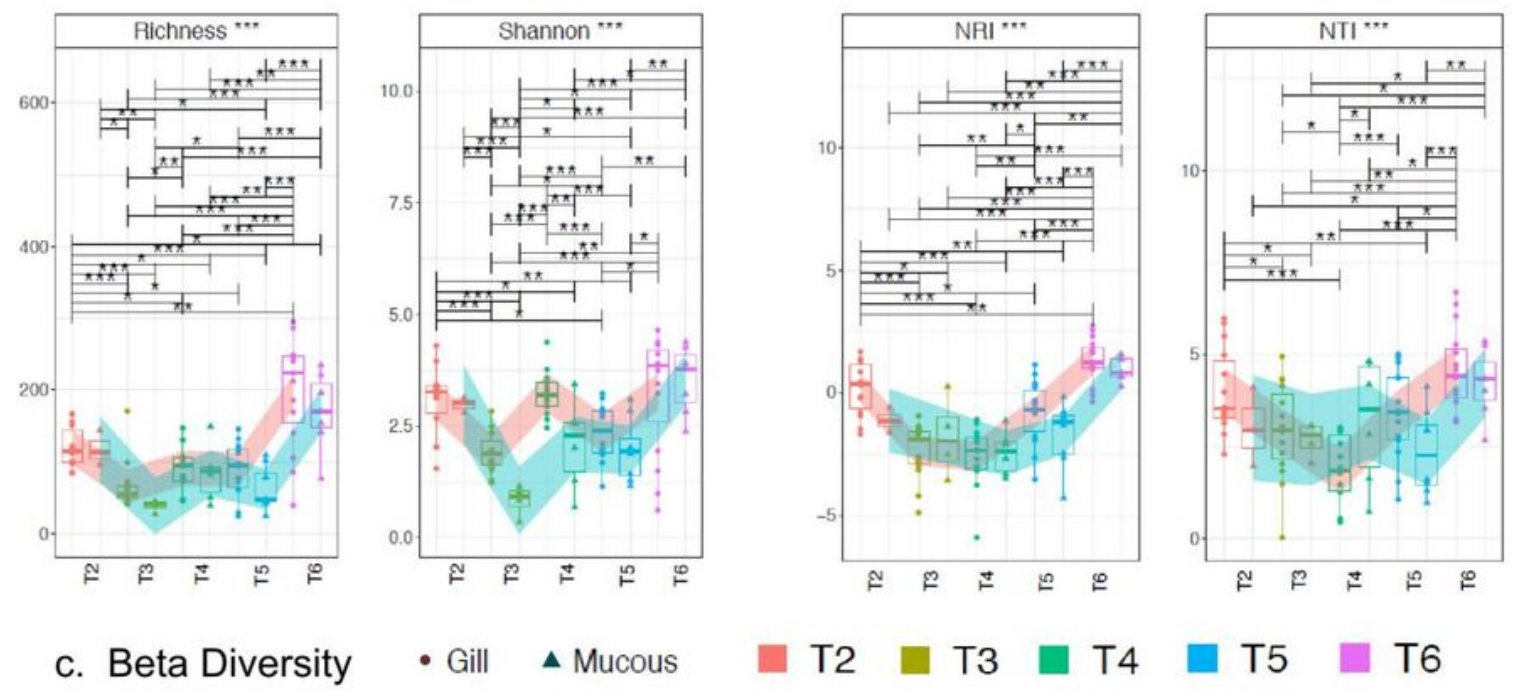

- Gill $\Delta$ Mucous

$\mathrm{T} 2$

T3 T4

T5

T6
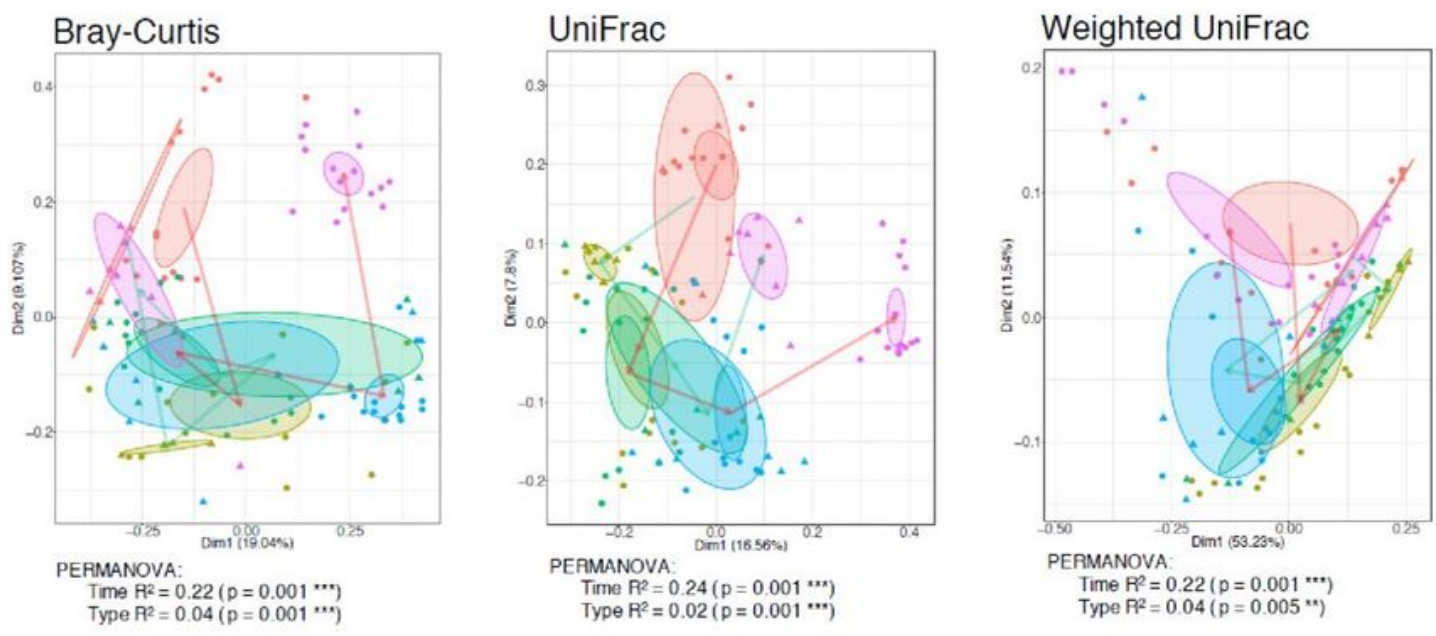

\section{d. Top 25 Abundant OTUs}

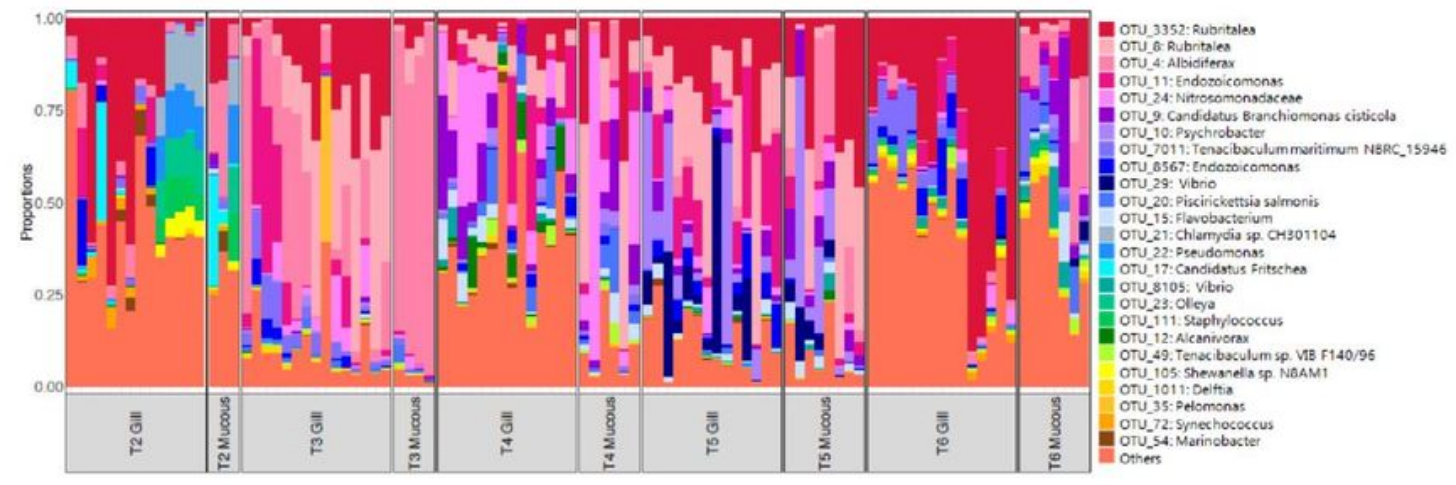

Figure 5

Microbial diversity and community structure from for cross-sectional 'gill vs mucous' analyses. (a), (b) and (c) represent alpha diversity, environmental filtering, and beta di-versity indices, respectively. In (a) 
and (b), that LOESS smoothing was performed sep-arately on gill and mucous samples, and PERMANOVA now considers both Time and Type parameters. In (c), ellipses are drawn at $95 \%$ confidence interval of standard error with lines from T2 to T6 plotted at the mean of the ordination values. (d) shows com-munity structure based on relative abundance of the top25 most abundant OTUs; lines for figures (a) and (b) connect two categories where the differences were significant (ANOVA) with * $(P<0.05)$, ** $(P<$ $0.01)$, or $* \star \star ~(p<0.001)$.

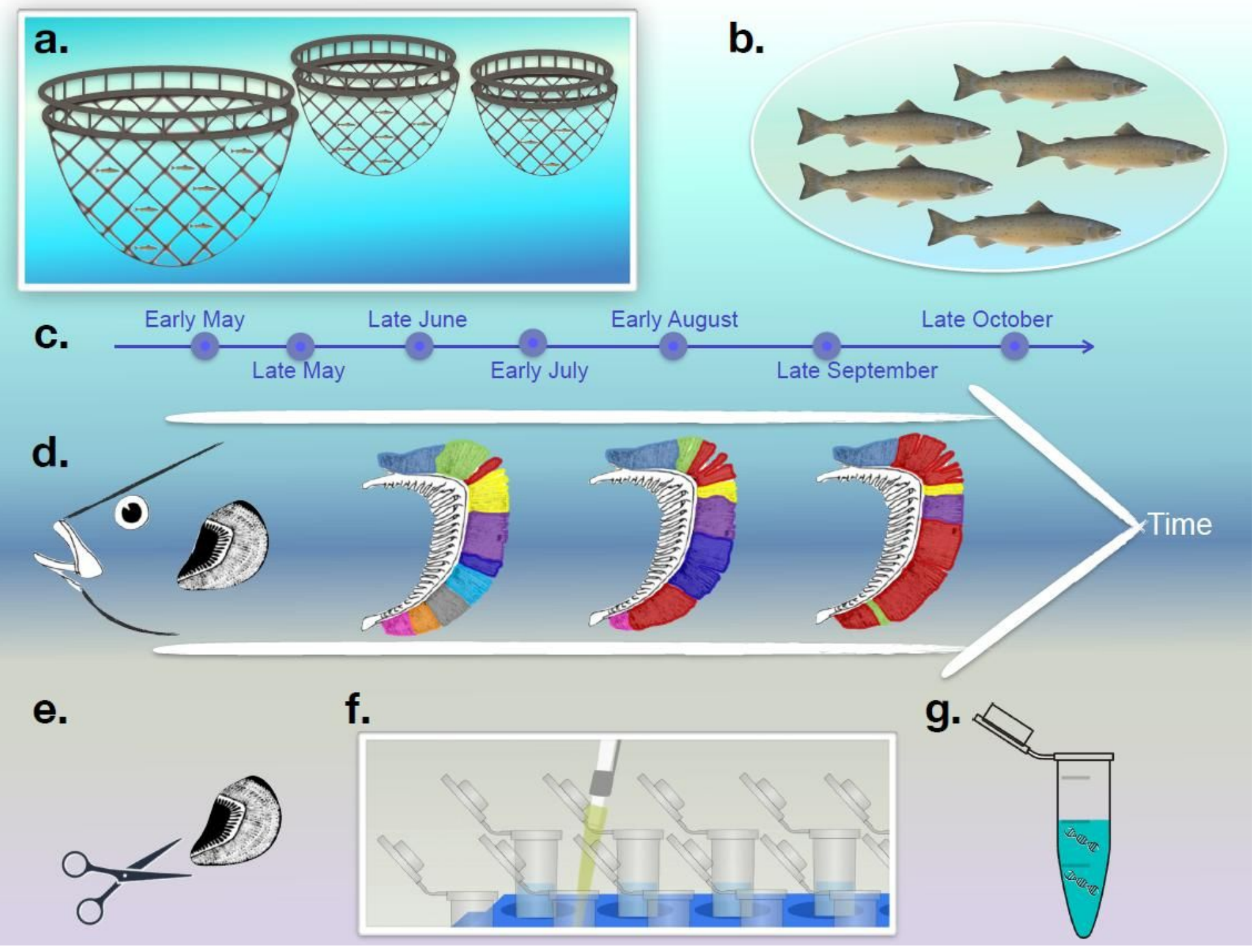

\section{Figure 6}

Overview of the sampling campaign and analytical approach. (a) Three cages were sampled to retrieve (b) five fish from each $(n=15)$ at each of (c) seven timepoints $(n=105)$ from May through October. (d) The hypothesis underpinning the study was that the amoebic gill disease (AGD) episode and abundance of $\mathrm{N}$. perurans (red shading) would be associated with a shifting microbiome. The analytical approach included $€$ sampling of gill and mucous samples, (f) a newly ptimized DNA extraction protocol incorporating several successive washes, and (g) downstream analyses of nucleic ac-ids.

\section{Supplementary Files}


This is a list of supplementary files associated with this preprint. Click to download.

- BirlangaetalLettertoEditorAnimalMicrobiome.pdf

- BirlangaetalSupplementaryMethodsFiguresTables.docx

- BirlangaetalSupplementaryDataEnvironmentalParameters.xlsx

- BirlangaetalSupplementaryDatasequences.xls 